\title{
ULTRAFAST DYNAMICS AND QUANTUM TRANSPORT OF ELECTRONS IN STRONGLY DISORDERED SEMICONDUCTORS
}

\author{
A. Kalvová and B. VelickÝ \\ Inst. of Phys., Czech. Acad. Sci. \\ Na Slovance 2, 18040 Praha 8, Czech Republic
}

\begin{abstract}
The new experiments on the response of electrons in semiconductors to femtosecond optical pulses call for developing adequate theoretical tools. A promising approach has been found in using the non-equilibrium Green functions approximately factorized on the basis of the so-called generalized Kadanoff-Baym ansatz. The present work investigates the validity of such approach on an example of a semiconductor with an alloy scattering, where the coherent potential approximation allows to construct the non-equilibrium Green functions directly, so that an explicit comparison with the ansatz decoupling is possible. The ansatz for the electron distribution is in this case justified as far as the quasiparticle picture for the individual electrons is appropriate.
\end{abstract}

PACS numbers: 72.10.-d, 78.47.+p, 71.25.-s

\section{Introduction}

Very short time non-equilibrium dynamics of electrons produced by strong external fields (optical pulses or high electric fields) in semiconductors take place in the time span before the reduced set of "robust" quasiparticle variables begins to emerge. In this regime, the time is so short that the picture of completed energy conserving collision breaks down. The standard Boltzmann equation description of the quasiparticle distribution disturbed by instantaneous collisions fails. A more complete description is necessary, including effects of quantum coherence, such as an interference of external fields with the internal dynamics of the system. During such short time internal quantum kinetics becomes non-Markovian, and the memory effects are important. This was demonstrated on a modulated decay of transient four-wave mixing in an experiment on the exciton-LO phonon scattering [1]. 
The non-equilibrium Green functions (NGF) are an elaborate systematic approach to treat electrons driven out of equilibrium on arbitrary time scales. The NGF equations of motion are not readily tractable, however, as the NGF have a double time structure. An important progress was achieved by introducing systematic ansatzes serving to express double time quantities by means of single-time structures like the non-equilibrium electron distribution. Well known is the generalized Kadanoff-Baym Ansatz (GKBA), leading for short times to the generalized master equation [2], and being fairly successfully applied. In this contribution, we test this GKBA against a model soluble directly for the NGF. Such model of a non-equilibrium transient, involving only alloy scattering, treated in the coherent potential approximation (CPA) was introduced in $[3,4]$.

\section{Notes about NGF}

Following [3], the electrons will be non-interacting fermions and a static random potential will be the only source of relaxation. For one sample configuration, the electron dynamics is controlled by a random one-electron Hamiltonian, and the evolution of the system is unitary with full preservation of the quantum coherence as contained in the one-electron retarded and advanced propagators $\left(\mathcal{G}^{\mathbf{r}}\left(t, t^{\prime}\right), \mathcal{G}^{\mathrm{a}}\left(t, t^{\prime}\right)\right)$. The one-electron density matrix evolves as

$$
\mathcal{R}(t)=\hbar^{2} \mathcal{G}^{\mathrm{r}}\left(t, t_{0}\right) \rho_{0} \mathcal{G}^{\mathrm{a}}\left(t_{0}, t\right)
$$

from its initial condition $\rho_{0}$ at the onset of the transient, $t=t_{0}$. The script letters denote quantities related to one configuration. This is obtained from the equal time limit of the particle GF,

$$
\mathcal{G}^{<}\left(t, t^{\prime}\right)=\mathrm{i} \hbar \mathcal{G}^{\mathrm{r}}\left(t, t_{0}\right) \rho_{0} \mathcal{G}^{\mathrm{a}}\left(t_{0}, t^{\prime}\right)
$$

Two key relations, apparent by inspection, hold in the unitary case. The propagators obey the semigroup property

$$
\mathcal{G}^{\mathrm{r}}\left(t, t^{\prime}\right)=\mathrm{i} \hbar \mathcal{G}^{\mathrm{r}}\left(t, t^{\prime \prime}\right) \mathcal{G}^{\mathrm{r}}\left(t^{\prime \prime}, t^{\prime}\right) \text { if } t>t^{\prime \prime}>t^{\prime}
$$

and for the particle function we get

$$
\mathcal{G}^{<}\left(t, t^{\prime}\right)=\mathcal{R}(t) \mathcal{G}^{\mathrm{a}}\left(t, t^{\prime}\right)-\mathcal{G}^{\mathrm{r}}\left(t, t^{\prime}\right) \mathcal{R}\left(t^{\prime}\right)
$$

This factorization is just the GKBA which thus appears as exact in the unitary case. We will be interested in the ensemble (configuration) averages, which evolve in a non-unitary way and manifest relaxation. The factorization of GKBA (4) will not be trivial then: the averages of the products depend on the correlations between the factors. For the averaged quantities we will use italics, $G^{\mathrm{r}}=\left\langle\mathcal{G}^{\mathrm{r}}\right\rangle, G^{\mathrm{a}}=$ $\left\langle\mathcal{G}^{\mathrm{a}}\right\rangle, G^{<}=\left\langle\mathcal{G}^{<}\right\rangle$. To obtain the equations for NGF, we configuration average the equations for $\mathcal{G}^{r, a}$, introduce the self-energies and get the Dyson equation for matrices $\boldsymbol{G}, \boldsymbol{\Sigma}$

$$
\mathrm{i} \hbar \partial_{t} \boldsymbol{G}-\langle H(t)\rangle \mathbf{1}-\boldsymbol{\Sigma} \boldsymbol{G}=\delta\left(t-t^{\prime}\right) \mathbf{1}
$$

where

$$
\boldsymbol{G}=\left(\begin{array}{cc}
G^{\mathrm{r}} & G^{<} \\
0 & G^{\mathrm{a}}
\end{array}\right), \quad \boldsymbol{\Sigma}=\left(\begin{array}{cc}
\Sigma^{\mathrm{r}} & \Sigma^{<} \\
0 & \Sigma^{\mathrm{a}}
\end{array}\right)
$$


All multiplications involve a matrix multiplication, an operator multiplication, and a time integration. To get $G^{<}$, we must convert the identities (6) into closed equations of motion. To achieve this, we have to make a physical approximation by expressing $\boldsymbol{\Sigma}$ as an approximate functional of $\boldsymbol{G}$. This is accomplished using the Langreth rule [5]: approximations for equilibrium such as the CPA, may be consistently transferred to the NGF by the correspondence $G^{\mathrm{r}} \rightarrow G, \Sigma^{\mathrm{r}} \rightarrow \Sigma$, etc.

\section{The model}

We select a two-band IIamiltonian, in which the site diagonal random potential acts only within the conduction band. The strong classical quasimonochromatic light wave field is coupled by a non-random dipole interaction, and treated in the rotating wave approximation (RWA). Two simplifications, a sudden step-like onset of the electromagnetic disturbance and a non-random initial condition (at $t=t_{0}$, all electrons are in valence band), make the model soluble using the CPA. For $t \geq t_{0}$ (at $t_{0}$ sudden onset of strong field), unitary transformation (see [6]) brings the IIamiltonian to a time-independent form,

$$
I I=\tilde{W}_{\mathrm{c}}+\mathcal{D}_{\mathrm{c}}+\tilde{W}_{\mathrm{v}}+\tilde{U}(\ell)
$$

where $\tilde{W}_{\mathrm{c}}$ describes the virtual crystal conduction band, $\tilde{W}_{\mathrm{v}}$ means the valence band shifted by the photon energy $h \omega$ (greater than the gap), $\mathcal{D}_{\mathrm{c}}$ is the random potential. Iere $\tilde{U}(t)=(e / m) A\left(p_{\mathrm{cv}}+p_{\mathrm{vc}}\right)$ is the interband dipole coupling, which acts as an effective band hybridization. $\Lambda$ dynamical optical gap is created [7], and both bands are mixed.

The static disorder acts as purely elastic scattering. This has the important consequences. First, the propagators $G^{\mathrm{r}}, G^{\mathrm{a}}$ are decoupled from $G^{<}$, and can be determined beforehand. Second, the configuration average is local in time so that, for times later than $t_{0}, G^{\mathrm{r}, \mathrm{a}}\left(t, t^{\prime}\right)=G^{r, \mathrm{a}}\left(t-t^{\prime}\right)$.

The particle double-time correlation function for $t>t_{0}, t^{\prime}>t_{0}$ has then the form

$$
\begin{aligned}
& G^{<}\left(t, t^{\prime}\right)=\mathrm{i} \hbar G^{\mathrm{r}}\left(t-t_{0}\right) \rho_{0} G^{\mathrm{a}}\left(t_{0}-t^{\prime}\right) \\
& \quad+\iint \mathrm{d} t_{1} \mathrm{~d} t_{2} G^{\mathbf{r}}\left(t-t_{1}\right) \Sigma^{<}\left(t_{1}, t_{2}\right) G^{\mathrm{a}}\left(t_{2}-t^{\prime}\right)
\end{aligned}
$$

The coherent transient will decay with the time (the initial condition is forgotten), the second term describes the gradual onset of an incoherent backflow. It provides the particle number conservation. The equation was solved in $[3,4]$ using Fourier transformation and calculating $\Sigma^{<}(z)(z$ is complex energy) by means of the usual CPA (see [8]).

\section{Testing the GKBA}

Here, we can give just a glimpse into the ansatz testing. Its validity is uneven in the Brillouin zone. In Fig. 1, we select a moderate situation and plot $G^{<}=$ $G^{<}\left(t_{1}, \tau=t_{1}-t_{2}\right)$. Exact $G^{<}$(full line) is compared with $G_{\mathrm{ANSATZ}}^{<}=\hbar G^{\mathrm{r}} \rho$ (dashed). For $\tau=0$, they trivially coincide. For $\tau=0.1 \mathrm{ps}$ ("large time") they come close together. During the quasiparticle formation, however, $\tau=0.01 \mathrm{ps}$, there emerges a substantial difference. Full test of its implications on integral quantities, like the transient polarization, is in progress. The plots given correspond [4] to the 

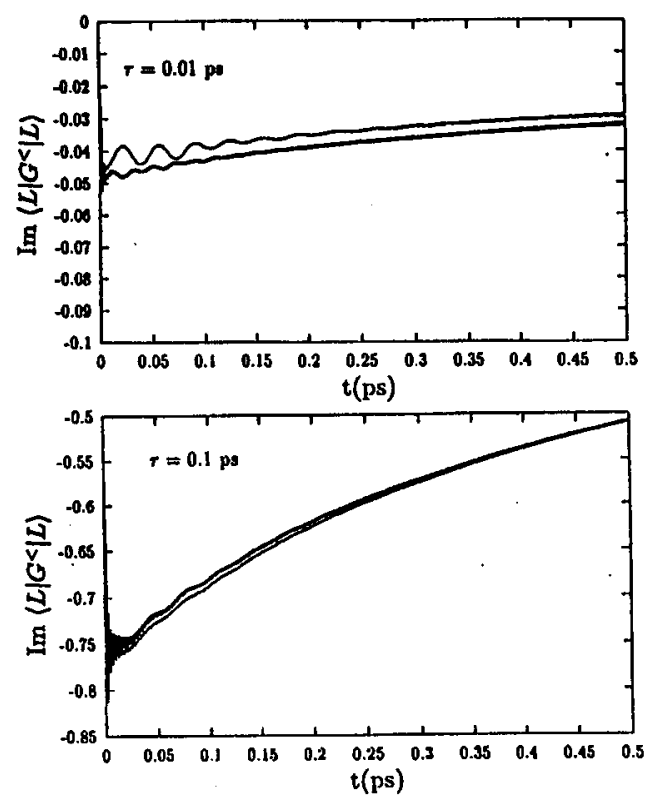

Fig. 1. Numerical test of GKBA for $L$-projection of the particle Green function $G^{<}$. The thin curve - exact $G^{<}$, the thick one $-G^{<}$found from relation (4), upper figure $-\tau=0.01 \mathrm{ps}$, lower one $-\tau=0.1 \mathrm{ps}$.

valence band like quasiparticle denoted by $L$, even more complex patterns appear in the "upper" quasiparticle band.

\section{References}

[1] L. Bányai, D.B. Tran Thoai, E. Retsamer, H. Haug, D. Steinbach, M.U. Wehner, M. Wegener, Phys. Rev. Lett., submitted.

[2] P. Lipavský, V. Śpička, B. Velický, Phys. Rev. B 34, 6933 (1986).

[3] A. Kalvová, B. Velický, Z. Phys. B 94, 273 (1994).

[4] B. Velický, A. Kalvová, Phys. Status Solidi B 188, 515 (1995).

[5] D. Langreth, J.W. Wilkins, Phys. Rev. B 6, 3189 (1972).

[6] V.M. Galitskii, S.P. Goreslavskii, V.F. Elesin, Sov. Phys.-JETP 30, 117 (1970).

[7] B. Velický, A. Pieczonková, Phys. Scr. Vol. T 19, 558 (1987).

[8] B. Velický, Phys. Rev. 184, 614 (1969). 